\title{
Golgi phosphoprotein 3 promotes the proliferation of gallbladder carcinoma cells via regulation of the NLRP3 inflammasome
}

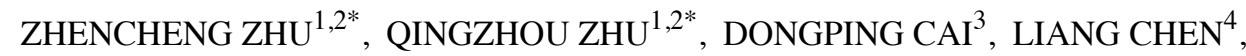 \\ WEIXUAN XIE ${ }^{2}$, YANG BAI ${ }^{2}$ and KUNLUN LUO ${ }^{1,2}$ \\ ${ }^{1}$ Anhui Medical University, Hefei, Anhui 230032; Departments of ${ }^{2}$ Hepatobiliary Surgery, ${ }^{3}$ Laboratory and \\ ${ }^{4}$ Cardiology, The 904th Hospital of Joint Logistic Support Force of PLA, Wuxi, Jiangsu 214044, P.R. China
}

Received January 18, 2021; Accepted April 2, 2021

DOI: $10.3892 /$ or.2021.8064

\begin{abstract}
Golgi phosphoprotein 3 (GOLPH3) has been demonstrated to promote tumor progression in various gastrointestinal malignancies. However, its effects in gallbladder carcinoma (GBC) remain unknown. In the present study, the expression levels of GOLPH3 and nucleotide-binding domain leucine-rich repeat and pyrin domain containing receptor 3 (NLRP3) in human GBC tissues were detected by immunohistochemistry, and the clinical data and survival of these patients were analyzed. Next, whether GOLPH3 could affect tumor proliferation via regulation of the NLRP3 inflammasome was investigated in vitro. The results demonstrated that GOLPH3 could promote GBC cell proliferation, and that it regulated protein expression levels of NLRP3, as well as Caspase-1 P10. Conversely, knockdown of NLRP3 reversed the effects of GOLPH3 overexpression on GBC cell proliferation. GOLPH3 and NLRP3 expression levels were found to be upregulated in GBC tissues and their expression was positively correlated. The expression of GOLPH3 and NLRP3 was associated with the expression of the proliferative marker Ki-67 in tissues, and associated with poor survival, tumor stage, degree of differentiation, depth of invasion, carbohydrate antigen 19-9 and C-reactive protein levels in patients with GBC. In summary, these results indicate that GOLPH 3 promotes GBC cell proliferation via a NLRP3/Caspase-1 pathway. GOLPH3 and NLRP3 participate in the process of human GBC growth and may serve as a potential therapeutic targets.
\end{abstract}

Correspondence to: Professor Kunlun Luo, Department of Hepatobiliary Surgery, The 904th Hospital of Joint Logistic Support Force of PLA, 101 Xingyuan North Road, Wuxi, Jiangsu 214044, P.R. China

E-mail:1k1197041@163.com

${ }^{*}$ Contributed equally

Key words: gallbladder carcinoma, molecular mechanism, inflammation, golgi phosphoprotein 3, nucleotide-binding domain leucine-rich repeat and pyrin domain containing receptor 3

\section{Introduction}

Gallbladder carcinoma (GBC) is a highly malignant tumor of the biliary system with a median survival time of only 6 months (1-3). The primary pathological type of GBC observed in patients is adenocarcinoma. The effects of current chemotherapeutic regimens are not sufficient for GBC due to the lack of effective drugs, making it particularly difficult to control the mortality rate of $\operatorname{GBC}(3,4)$. Due to the close relationship between inflammation and GBC, investigation of inflammatory-related molecular mechanisms may highlight novel specific targets for the treatment of GBC (4).

Golgi phosphoprotein 3 (GOLPH3) is a component of the trans Golgi network (TGN) (5-7). Physiologically, GOLPH3 stabilizes the Golgi structure and promotes the sprouting of Golgi vesicles through binding with phosphatidylinositol 4-phosphate [PtdIns(4)P] and myosin18A $(5,8,9)$. Excessive activation of GOLPH3 induces the breakdown of the Golgi apparatus (8-10). Recently, several studies have reported that GOLPH3 serves an important role in the progression of several types of cancer, such as hepatocellular carcinoma, gastric adenocarcinoma and lung adenocarcinoma (11-14). In addition, GOLPH3 is involved in the process of chemotherapeutic resistance, further highlighting its close relationship with tumor progression $(8,15)$. It has been reported that GOLPH3 promotes tumor growth via several signaling pathways, including the PI3K/AKT/mTOR pathway (14-17). However, the role and mechanism of GOLPH3 in GBC remain unknown.

Nucleotide-binding domain leucine-rich repeat (NLR) and pyrin domain containing receptor 3 (NLRP3) comprise the NLRP3 inflammasomes together with apoptosis associated speck like protein and pro-caspase-1 $(18,19)$. Physiological levels of NLRP3 activation protects the body's inflammatory immune system via activation of Caspase-1, whereas uncontrolled activation will lead to dysregulated inflammation, autoimmune diseases, neurodegenerative diseases, and even malignant tumors (20-24). Recently, a number of studies have reported that the overactivation of NLRP3 is closely associated with the progression of several malignant tumors (24-27). To date, there are numerous studies on the downstream effects of NLRP3 (22-25). There is evidence that the NLRP3/Caspase-1 pathway can promote the occurrence and progression of adenocarcinoma, and IL-1 $\beta$, an important member of the NLRP3 
inflammasome, has been found to be closely related with the proliferation of GBC $(25,28)$. Nevertheless, the specific molecular mechanisms by which NLRP3 is pathophysiologically activated remains unknown.

Thus, both GOLPH3 and NLRP3 are associated with tumor progression. However, the relationship between GOLPH3 and NLRP3 in tumorigenesis and progression remains unclear. Evidence has shown that excessive activation of GOLPH3 can cause the fragmentation of the Golgi apparatus, and the fragmentation of the TGN is closely associated with the activation of NLRP3 $(8,10,21)$. In addition, PtdIns(4)p is required when NLRP3 is activated, of which, the free amount of PtdIns(4)p is associated with GOLPH3 $(5,21,29)$. Moreover, there is evidence that mTOR can affect the activation of NLRP3 inflammasomes by regulating reactive oxygen species, and the activation of mTOR is also largely regulated by GOLPH3 $(6,30)$. Additionally, GOLPH3 and NLRP3 have been reported to both be regulated by the same upstream protein PD2 $(6,31)$. Therefore, it is hypothesized that GOLPH3 may be an upstream factor of NLRP3. In the present study, the expression levels of GOLPH3 and NLRP3 in human GBC tissues were detected via immunohistochemistry, and the clinical data and survival of these patients were analyzed. Then, it was tested whether GOLPH3 can affect tumor proliferation via regulation of the NLRP3 inflammasome in vitro. Through these experiments, the aim was to find out whether GOLPH3 is an upstream regulator of the NLRP3/Caspase-1 pathway in the proliferation of GBC cells.

\section{Materials and methods}

Cell culture. The human GBC-SD cell line was purchased from Guangzhou Cellcook Biotech Co., Ltd. Cells were grown in RPMI-1640 medium (Hyclone; Cytiva) supplemented with 10\% FBS (Gibco; Thermo Fisher Scientific, Inc.) in a humidified incubator with $5 \% \mathrm{CO}_{2}$ at $37^{\circ} \mathrm{C}$.

Small interfering (si)RNA and plasmid transfection. Knocking down of GOLPH3 and NLRP3 was performed using specific siRNA Oligos (Guangzhou RiboBio Co., Ltd.). The sequences of the siRNAs were as follows: si-negative control (NC) sense, 5'-UUCUCCGAACGUGUCACGU-3' and anti-sense, 5'-ACG UGACACGUUCGGAGAA-3'; si-NLRP3 (NLRP3-SiR) sense, 5'-GGCAGACCAUGUGGAUCUATT-3' and anti-sense, 5'-UAGAUCCACAUGGUCUGCCTT-3'; si-GOLPH3-A (GOLPH3-SiA) sense, 5'-GGUGAGACAUGGAAUCCAU-3' and anti-sense, 5'-AUGGAUUCCAUGUCUCACC-3'; and si-GOLPH3-B (GOLPH3-SiB) sense, 5'-GCAGCGCCUCAU CAAGAAA-3' and anti-sense, 5'-UUUCUUGAUGAGGCG CUGC-3'.

Lipofectamine ${ }^{\circledR} 2000$ (Invitrogen; ThermoFisherScientific, Inc.) was used for transfection. A total of 1 day before transfection, $3.5 \times 10^{5}$ cells were plated in a 6 -well plate, such that they were $60-70 \%$ confluent at the time of transfection. Then, $30 \mathrm{nmol}$ of the specific siRNA and $5 \mu \mathrm{l}$ Lipofectamine 2000 reagent was mixed with $250 \mu 1$ Opti-MEM (Gibco; Thermo Fisher Scientific, Inc.), and left to stand for $5 \mathrm{~min}$. The diluted siRNA and Lipofectamine 2000 reagent were combined and incubated for another $20 \mathrm{~min}$. Subsequently, the mixture was added to the cells. All siRNA silencing experiments were performed three times independently. siNC was used as the control group.
Additionally, treatment with Lipofectamine 2000 alone, under equivalent culture conditions, was used as a blank control group (Mock). All transfections lasted for $6 \mathrm{~h}$ in a humidified incubator with $5 \% \mathrm{CO}_{2}$ at $37^{\circ} \mathrm{C}$. Then, $48 \mathrm{~h}$ after transfection, subsequent experimentation was performed.

GOLPH3 was overexpressed using a GOLPH3 pcDNA-3xFlag-C plasmid (Guangzhou RiboBio Co., Ltd.). An empty plasmid (3xFlag) was used as the negative control group. Treatment with Lipofectamine 2000 alone, under equivalent culture conditions, was used as a blank control group (Mock). The transfection protocol of the overexpression plasmid was similar to that of the siRNA, except that $2 \mu \mathrm{g}$ plasmid was used for overexpression.

For co-transfections, cells were divided into the following five groups: i) GOLPH3 + NLRP3-SiNC group, GOLPH3 pcDNA-3xFlag-C plasmid and si-NC; ii) GOLPH3 + NLRP3-SiR group, GOLPH3 plasmid and si-NLRP3; iii) 3xFlag + NLRP3-SiR group, empty plasmid (3xFlag) and si-NLRP3; iv) 3xFlag + NLRP3-SiNC group (negative control), empty plasmid (3xFlag) and siNC; and v) mock group (blank control), treatment with Lipofectamine 2000 alone under equivalent culture conditions. The transfection protocol of the co-transfection was similar to that of the siRNA and the overexpression plasmid, except that $1 \mu \mathrm{g}$ plasmid was used for co-transfection.

EdU incorporation assay. Transfected cells were seeded into 96 -well plates at $5 \times 10^{4}$ cells per well and incubated for $24 \mathrm{~h}$. When the confluency reached $60-70 \%$, transfection was performed as described above. After $48 \mathrm{~h}$, the cells were cultured in EdU medium for $2 \mathrm{~h}$ (Guangzhou RiboBio Co., Ltd.). Then, the cells were fixed with $4 \%$ paraformaldehyde for $30 \mathrm{~min}$ at room temperature, and treated with $0.5 \%$ Triton X-100 for $10 \mathrm{~min}$ at room temperature. After washing with PBS for 5 min with constant shaking, the cells were treated with $100 \mu \mathrm{l} 1 \mathrm{X}$ Apollo ${ }^{\circledR}$ reaction cocktail (Guangzhou RiboBio Co., Ltd.) for $30 \mathrm{~min}$. The DNA content of cells were stained with $100 \mu \mathrm{l}$ of Hoechst $33342(5 \mu \mathrm{g} / \mathrm{ml})$ for another $30 \mathrm{~min}$ at room temperature. The results were observed under a fluorescence microscope (magnification, x200, Olympus DP80; Olympus Corporation).

Cell viability assay. Cell viability was measured using a Cell Counting Kit-8 (CCK-8) assay (Dojindo Molecular Technologies, Inc.) three times independently. Cells $\left(3.5 \times 10^{4}\right)$ were plated in a 96-well plate and cultured for $24 \mathrm{~h}$, at $50-60 \%$ confluence at the time of transfection, and then transfected with siRNA or plasmid for $48 \mathrm{~h}$. A total of $10 \mu \mathrm{l}$ CCK-8 solution was added to each well and incubated for a further $2 \mathrm{~h}$. The absorbance at $450 \mathrm{~nm}$ (OD450) was measured using a microplate reader (Autobio Diagnostics Co., Ltd.).

Western blotting. The cells were lysed in RIPA lysis buffer (Beijing Solarbio Science \& Technology Co., Ltd.) for $20 \mathrm{~min}$ on ice and collected using a cell scraper (Corning, Inc.). The total protein concentration was determined using a BCA Protein assay kit (Beijing Solarbio Science \& Technology Co., Ltd.). Equivalent amounts of protein $(40 \mu \mathrm{g})$ were loaded per lane to a $10 \%$ gel, resolved via SDS-PAGE, and subsequently transferred to a PVDF membrane. The membrane was blocked using $3 \%$ BSA (Beijing Solarbio Science \& Technology Co., Ltd.) or 
$5 \%$ skimmed milk in TBS with $0.05 \%$ Tween-20 (TBST) for $1 \mathrm{~h}$ with constant shaking at room temperature. The membranes were incubated with antibodies against GOLPH3 (1:2,000; cat. no. ab98023; Abcam), NLRP3 (1:1,000; cat. no. ab263899; Abcam), Caspase-1/P10 (1:1,000; cat. no. 22915-1-AP; ProteinTech Group, Inc.), IL-1 $\beta$ (1:1,000; cat. no. ab9722; Abcam), IкB (1:1,000; cat. no. ab76429; Abcam), phosphorylated (p)-IкB (1:10,000; cat. no. ab133462; Abcam) and $\beta$-actin (1:5,000; cat. no. 66009-1-Ig; ProteinTech Group, Inc.) at $4^{\circ} \mathrm{C}$ overnight, and then incubated with horseradish peroxidase (HRP)-conjugated goat anti-mouse (cat. no. SA00001-1) and goat anti-rabbit (cat. no. SA00001-2) secondary antibodies (all at 1:10,000; ProteinTech Group, Inc.) for $1 \mathrm{~h}$ at room temperature after washing with TBST. The bands were visualized using a chemiluminescence detection kit (EMD Millipore) and densitometry was performed using ImageJ version 1.8.0.112 (National Institutes of Health). The relative amount of protein was normalized by dividing the density value of the target protein by the density value of the internal loading control.

\section{Immunohistochemistry}

Specimen acquisition. Pathological sections of gallbladders that had previously been paraffin-embedded pathological sections from 25 patients with GBC that underwent radical resection of GBC (GBC group) between January 2016 and January 2018 at the 904th Hospital of Joint Logistic Support Force of PLA (Wuxi, China), as well as 10 patients with gallstones who underwent laparoscopic cholecystectomy during the same period (control group) were obtained. Patients with GBC were diagnosed with T1-3 gallbladder adenocarcinoma by preoperative imaging, intraoperative exploration and postoperative pathology, and there was no evidence of distant metastases in any of the cases. The histopathology of the gallbladder in patients with gallstones after surgery suggested cholecystitis. All specimens were provided by the Department of Pathology, The 904th Hospital of Joint Logistic Support Force of PLA (25 gallbladder adenocarcinoma tissues, 10 gallbladder tissues with cholecystitis). Written informed consent was obtained from all patients and the study was approved by the Ethics Committee of the 904th Hospital of Joint Logistic Support Force of PLA (approval no. 2019-10-006).

Tissue staining. Tissue staining was performed using an immunohistochemical S-P kit (OriGene Technologies, Inc.), according to the manufacturer's protocol. Briefly, $4 \%$ paraformaldehyde was used for fixation at $4^{\circ} \mathrm{C}$ for $24 \mathrm{~h} .5 \%$ BSA (Beijing Solarbio Science \& Technology Co., Ltd.) was used to block non-specific sites for $30 \mathrm{~min}$ at $37^{\circ} \mathrm{C} .3 \%$ hydrogen peroxide was used to block endogenous peroxidase activity at room temperature for $10 \mathrm{~min}$ to reduce non-specific background staining. The $4-\mu \mathrm{m}$ sections were incubated with GOLPH3 (1:100; cat. no. 19112-1-AP; ProteinTech Group, Inc.), NLRP3 (1:50; cat. no. 19771-1-AP; ProteinTech Group, Inc.) or Ki-67 antibodies (1:5,000; cat. no. 27309-1-AP; ProteinTech Group, Inc.) with PBS as the blank control group) for $2 \mathrm{~h}$ at $37^{\circ} \mathrm{C}$, followed by incubation with biotin-conjugated goat anti-rabbit IgG antibody (1:200; cat. no. TA130016; OriGene Technologies, Inc.) for $1 \mathrm{~h}$ at room temperature. Then, sections were reacted with 3 , 3'-diaminobenzidine chromogenic reagent. The sections were counterstained with hematoxylin at room temperature for $30 \mathrm{sec}$ and immersed in graded series of alcohol solutions, then dehydrated in $100 \%$ xylene and finally sealed with neutral glue. The results were observed under an Olympus CX23 inverted microscope (Olympus Corporation).

Evaluation. The results of immunohistochemistry were analyzed using semi-quantitative integration (32). Randomly, five fields of view were selected for each slice (magnification, $\mathrm{x} 200$ ), and two pathologists assessed the degree of staining. Staining intensity (SI) was scored as follows: i) 0 points, undyed; ii) 1 point, lightly dyed or light yellow; iii) 2 points, yellow; and iv) 3 points, darkly dyed, brown or tan. The percentage of positively stained cells (PP) was scored as follows: i) 0 points, no staining; ii) 1 point, $\leq 10 \%$ of cells stained; iii) 2 points, $11-50 \%$ of cells stained; iv) 3 points, $51-80 \%$ of cells stained; and v) 4 points, $>80 \%$ of cells stained. The final score was the product of the SI and the PP score: $0-3$ points, negative (-) and $>3$ points, positive (+).

Patients. The clinical data of the 35 patients (25 GBC cases, 10 gallstone cases) were collected. The 25 patients with GBC (GBC group) all underwent $\mathrm{R} 0$ resection, including 10 male patients and 15 female patients, with a median age of 65.0 years (range, 36-85 years). The tumor staging was evaluated according to the 8th Edition of the AJCC TNM staging system (33); amongst the patients with GBC, seven cases were T1 stage, eight cases were T2 stage and 10 cases were T3 stage. In addition, 10 cases were accompanied by lymph node metastasis, none of which had distant metastases. The median survival of patients with GBC in the cohort was 19 months, and the 1, 3 and 5-year survival rates of the patients were 72, 24 and 12\%, respectively. Amongst the 10 patients with gallstones (Control), four were men and six were women, with a median age of 65.5 years (range, 50-74 years). There was no significant difference in the sex or age between the GBC and control groups $(\mathrm{P}>0.05)$. None of patients had received any targeted antitumor treatments before surgery, such as chemotherapy, radiotherapy or targeted therapy.

Surgery. All patients with GBC underwent radical resection of GBC, and postoperative pathology confirmed R0 resection. Radical resection included cholecystectomy, partial hepatectomy, or regional lymph node dissection (hepatoduodenal ligament + posterior pancreas + aortic root). The scope of surgical resection was based on the tumor stage, and whether the extrahepatic bile duct was removed was determined according to the degree of invasion of the bile duct. All patients with cholelithiasis underwent laparoscopic cholecystectomy.

Follow-up. After the operation, patients with GBC were regularly followed up through clinics and telephone calls. In the first year, the patients were followed up every 3 months, every 6 months in the second year, and every 12 months in the third year. The follow-up was performed until 30 September 2020. The time between surgery and death or the end of follow-up was considered the overall survival.

Statistical analysis. All data were analyzed using SPSS version 23 (IBM Corp.), and the experiments were repeated at least three times. Quantitative data were analyzed using a unpaired Student's t-test or a Mann-Whitney U test, and 


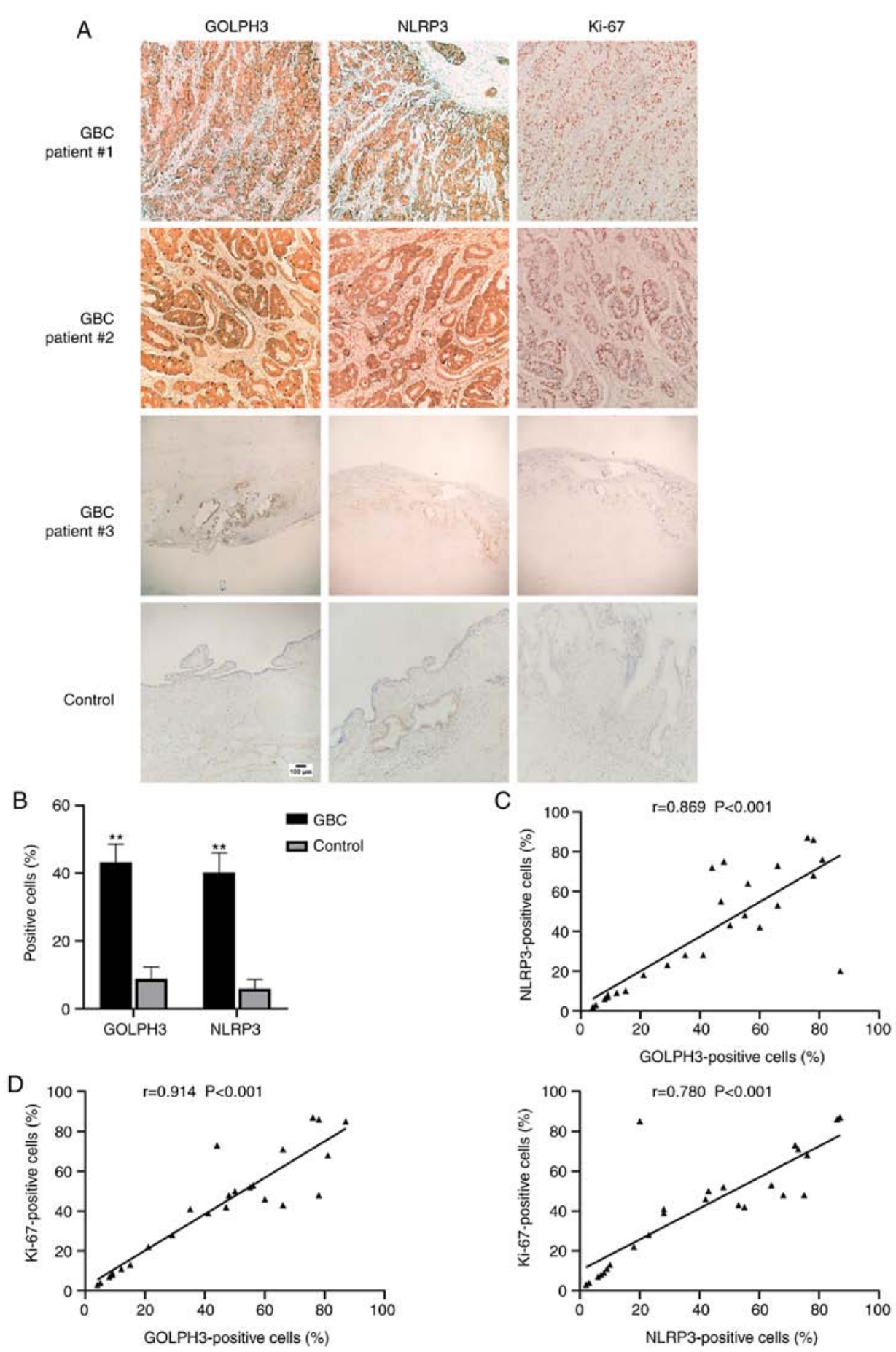

Figure 1. Expression of GOLPH3 and NLRP3 in human gallbladder tissue. (A) Representative images of GOLPH3, NLRP3 and Ki-67 in human GBC tissues and non-tumorous gallbladder tissues by immunohistochemistry. (B) Histogram showing the quantitative analysis of the percentage of GOLPH3- and NLRP3-positive cancer cells. (C) Correlation between the levels of GOLPH3 and NLRP3 in human GBC tissue ( $r=0.869 ; \mathrm{P}<0.001)$. (D) GOLPH3 and NLRP3 expression was associated with Ki-67 expression in human GBC tissues (GOLPH3, $\mathrm{r}=0.914, \mathrm{P}<0.001$; NLRP3, $\mathrm{r}=0.780$, $\mathrm{P}<0.001$ ). Data are expressed as the mean \pm standard error of the mean of three independent experiments. Scale bar, $100 \mu \mathrm{m}$. ${ }^{* *} \mathrm{P}<0.01$ vs. control. GOLPH3, Golgi phosphoprotein 3; GBC, gallbladder cancer; NLRP3, nucleotide-binding domain leucine-rich repeat and pyrin domain containing receptor 3.

are expressed as the mean \pm standard deviation, or the median (interquartile range), respectively. Qualitative data were analyzed using a $\chi^{2}$ test or a Fisher's exact test. The Kaplan-Meier method was used for survival analysis. The Log-rank test was used to analyze the Kaplan-Meier curves. Pearson's linear correlation analysis was used to assess correlations. $\mathrm{P}<0.05$ was considered to indicate a statistically significant difference.

\section{Results}

GOLPH3 and NLRP3 expression is upregulated in human GBC tissues and its expression is associated with Ki-67. First, the clinical relevance of GOLPH3 and NLRP3, as well as their relationship in clinical GBC samples were evaluated. The expression of GOLPH3, NLRP3 and the proliferative marker $\mathrm{Ki}-67$ (34) in the gallbladder tissues of the 25 patients with GBC 
Table I. Differences in the expression of GOLPH3 and NLRP3 in different gallbladder tissues.

\begin{tabular}{|c|c|c|c|c|c|}
\hline \multirow[b]{2}{*}{ Group } & \multirow[b]{2}{*}{$\mathrm{n}$} & \multicolumn{2}{|c|}{ GOLPH3 } & \multicolumn{2}{|c|}{ NLRP3 } \\
\hline & & Negative, n (\%) & Positive, n (\%) & Negative, n (\%) & Positive, n (\%) \\
\hline GBC & 25 & $9(36.0)$ & $16(64.0)$ & $10(40.0)$ & $15(60.0)$ \\
\hline Gallstones & 10 & $8(80.0)$ & $2(20.0)$ & $9(90.0)$ & $1(10.0)$ \\
\hline P-value & & 0.027 & & 0.010 & \\
\hline
\end{tabular}

GOLPH3, Golgi phosphoprotein 3; GBC, gallbladder cancer; NLRP3, nucleotide-binding domain leucine-rich repeat and pyrin domain containing receptor 3 .

and 10 patients with gallstones were assessed using immunohistochemical staining (Fig. 1). As shown in Fig. 1A and B, the percentage of GOLPH3- and NLRP3-positive cells in the gallbladder tissue of GBC cases was 4-5 times higher than that of the non-cancer tissues (GOLPH3 GBC, 43.2 $\pm 27.1 \%$ and Control, 8.8 $\pm 11.1 \%$; NLRP3 GBC, $40.2 \pm 29.0 \%$ and Control, $5.9 \pm 8.7 \%$ ), and the proportion of patients with GOLPH3- and NLRP3-positive expression in the GBC cases was significantly higher than that in the patients with gallstones (Table I). In addition, the percentage of GOLPH3-positive cells was positively correlated with the percentage of NLRP3-positive cells in GBC tissues (Fig. 1C). Additionally, the percentage of GOLPH3- and NLRP3-positive cells were both positively correlated with the percentage of Ki-67-positive cells in the GBC tissues (Fig. 1D). The results of the immunohistochemistry experiments also showed a similar trend (Fig. 1A).

Expression of GOLPH3 and NLRP3 is associated with a poor prognosis in patients with $G B C$. To explore the clinical significance of GOLPH3 and NLRP3, the clinicopathological data of the 25 patients with GBC was analyzed. The results showed that there was no significant difference in the age or sex of the GOLPH3- or NLRP3-positive and negative patients (Table II). However, the survival of GOLPH3-positive patients $(16 / 25)$ or NLRP3-positive patients $(15 / 25)$ was worse than the patients with negative expression (GOLPH3, median 16 vs. 55 months, $\mathrm{P}=0.001$; NLRP3, median 16 vs. 40 months, $\mathrm{P}<0.001$; Fig. 2A and B). Next, the relationship between the positive expression of GOLPH3 or NLRP3 and the clinicopathological characteristics of patients with GBC was assessed. The results suggested that GOLPH3 - or NLRP3-positive patients had more advanced tumors (the TNM stage), deeper infiltration, poorly differentiated tumors and higher carbohydrate antigen 19-9 (CA199) or C-reactive protein (CRP) levels (Table II).

GOLPH3 promotes the proliferation of human GBC cells. To explore the possible role of GOLPH3 in the development of human GBC, GOLPH3-specific siRNAs were used to knock down the expression of GOLPH3 in GBC-SD cells. As shown in Fig. 3A and B, compared with the GOLDPH3-SiNC group, the protein expression levels of GOLPH3 decreased by 55-65\% in the GBC-SD cells transfected with GOLDPH3-SiA and $-\mathrm{SiB}$. Next, cell proliferation after GOLPH3 knockdown was evaluated using EdU and cell viability was evaluated using CCK-8 assays. The results of the EdU assay showed that the

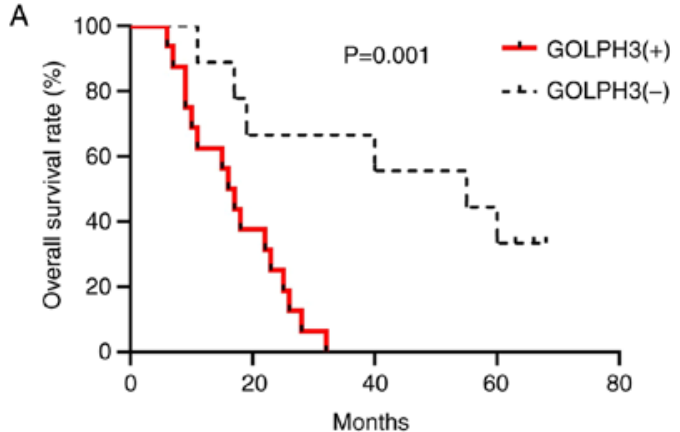

B

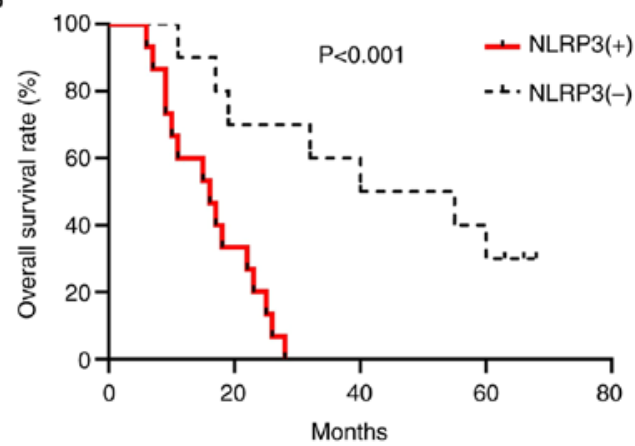

Figure 2. Survival analysis of the 25 patients with gallbladder cancer following surgery. (A) The postoperative survival of GOLPH3-positive patients was significantly worse than that of the negative patients $(\mathrm{P}=0.001)$. (B) The postoperative survival of the NLRP3-positive patients was worse than that of the negative patients $(\mathrm{P}<0.001)$. GOLPH3, Golgi phosphoprotein 3; NLRP3, nucleotide-binding domain leucine-rich repeat and pyrin domain containing receptor 3 .

EdU-positive cells of the GOLPH3-SiA and -SiB groups were reduced by 40-80\% compared with the GOLDPH3-SiNC group (Fig. 3C and D). Additionally, the results of the CCK-8 assay indicated that the OD450 in the GOLPH3-SiA and -SiB groups decreased by $20-30 \%$ compared with the GOLDPH3-SiNC group (Fig. 3E).

Next, GOLPH3 was overexpressed in GBC-SD cells, and the change in the proliferation of GBC cells was assessed. The results of western blotting showed that the GOLPH3-overexpression plasmid (Fig. 4F) was successfully transfected (Fig. 4A and B). The EdU assay showed that when GOLPH3 was overexpressed, the percentage of EdU positive cells increased by $30 \%$ compared with the $3 \mathrm{xFlag}$ group (Fig. 4C and D). In addition, the results of the CCK-8 assay suggested that the absorbance at $450 \mathrm{~nm}$ of the 
Table II. Clinicopathological characteristics of the 25 patients with GBC.

A, GOLPH3 expression

\begin{tabular}{|c|c|c|c|c|}
\hline Variable & Negative & Positive & $\chi^{2}$ & P-value \\
\hline Sex, n (\%) & & & 0.000 & 1.000 \\
\hline Male & $4(44.4)$ & $6(37.5)$ & & \\
\hline Female & $5(55.6)$ & $10(62.5)$ & & \\
\hline With gallstones, n (\%) & & & 0.405 & 0.602 \\
\hline Yes & $7(77.8)$ & $14(87.5)$ & & \\
\hline No & $2(22.2)$ & $2(12.5)$ & & \\
\hline With hypertension, n (\%) & & & 0.762 & 0.434 \\
\hline Yes & $5(55.6)$ & $6(37.5)$ & & \\
\hline No & $4(44.4)$ & $10(62.5)$ & & \\
\hline Bile duct invasion, $\mathrm{n}(\%)$ & & & 0.011 & 0.915 \\
\hline Yes & $3(33.3)$ & $5(31.3)$ & & \\
\hline No & $6(66.7)$ & $11(68.8)$ & & \\
\hline Infiltration depth, n (\%) & & & 4.890 & $0.040^{\mathrm{b}}$ \\
\hline $\mathrm{T} 1+\mathrm{T} 2$ & $8(88.9)$ & $7(43.8)$ & & \\
\hline $\mathrm{T} 3$ & $1(11.1)$ & $9(56.3)$ & & \\
\hline Lymph node metastasis, n (\%) & & & 1.852 & 0.229 \\
\hline Yes & $2(22.2)$ & $8(50.0)$ & & \\
\hline No & $7(77.8)$ & $8(50.0)$ & & \\
\hline Differentiation, n (\%) & & & 7.677 & $0.012^{\mathrm{b}}$ \\
\hline Poor & $4(44.4)$ & $15(93.8)$ & & \\
\hline High/medium & $5(55.6)$ & $1(6.3)$ & & \\
\hline TNM stage, $\mathrm{n}(\%)$ & & & 6.173 & $0.033^{\mathrm{b}}$ \\
\hline $\mathrm{I}+\mathrm{II}$ & $8(88.9)$ & $6(37.5)$ & & \\
\hline III & $1(11.1)$ & $10(62.5)$ & & \\
\hline Chemotherapy $^{\mathrm{a}}, \mathrm{n}(\%)$ & & & 0.043 & 0.835 \\
\hline Yes & $3(33.3)$ & $6(37.5)$ & & \\
\hline No & $6(66.7)$ & $10(62.5)$ & & \\
\hline Age, years, mean $\pm \mathrm{SD}$ & $63.89 \pm 7.47$ & $64.63 \pm 12.94$ & $-0.156^{c}$ & 0.878 \\
\hline White blood cells $\left(\times 10^{9} / 1\right)$, mean \pm SD & $6.40 \pm 1.58$ & $6.37 \pm 1.40$ & $0.061^{\mathrm{c}}$ & 0.951 \\
\hline Total bilirubin, $\mu \mathrm{mol} / \mathrm{l}$, median (interquartile range) & $14.20(10.20,19.05)$ & $21.65(12.00,139.68)$ & $94.000^{\mathrm{d}}$ & 0.229 \\
\hline $\mathrm{CRP}, \mathrm{g} / \mathrm{l}$, median (interquartile range) & $1.80(0.95,3.20)$ & $5.25(2.90,16.70)$ & $121.000^{\mathrm{d}}$ & $0.004^{\mathrm{b}}$ \\
\hline $\mathrm{CEA}, \mu \mathrm{g} / \mathrm{l}$, median (interquartile range) & $1.97(1.68,4.01)$ & $2.12(1.56,5.82)$ & $72.000^{\mathrm{d}}$ & 1.000 \\
\hline CA199, U/ml, median (interquartile range) & $10.99(9.71,26.32)$ & $71.74(20.98,423.59)$ & $108.000^{\mathrm{d}}$ & $0.043^{\mathrm{b}}$ \\
\hline
\end{tabular}

B, NLRP3 expression

\begin{tabular}{|c|c|c|c|c|}
\hline Variable & Negative & Positive & $\chi^{2}$ & P-value \\
\hline $\operatorname{Sex}, \mathrm{n}(\%)$ & & & 0.000 & 1.000 \\
\hline Male & $4(40.0)$ & $6(40.0)$ & & \\
\hline Female & $6(60.0)$ & $9(60.0)$ & & \\
\hline With gallstones, n (\%) & & & 0.000 & 1.000 \\
\hline Yes & $8(80.0)$ & $13(86.7)$ & & \\
\hline No & $2(20.0)$ & $2(13.3)$ & & \\
\hline With hypertension, $\mathrm{n}(\%)$ & & & 0.244 & 0.697 \\
\hline Yes & $5(50.0)$ & $6(40.0)$ & & \\
\hline No & $5(50.0)$ & $9(60.0)$ & & \\
\hline Bile duct invasion, $\mathrm{n}(\%)$ & & & 0.031 & 0.861 \\
\hline Yes & $3(30.0)$ & $5(33.3)$ & & \\
\hline No & $7(70.0)$ & $10(66.7)$ & & \\
\hline
\end{tabular}


Table II. Continued.

\begin{tabular}{|c|c|c|c|c|}
\hline \multicolumn{5}{|l|}{ B, NLRP3 expression } \\
\hline Variable & Negative & Positive & $\chi^{2}$ & P-value \\
\hline Infiltration depth, n (\%) & & & 6.250 & $0.018^{\mathrm{b}}$ \\
\hline $\mathrm{T} 1+\mathrm{T} 2$ & $9(90.0)$ & $6(40.0)$ & & \\
\hline $\mathrm{T} 3$ & $1(10.0)$ & $9(60.0)$ & & \\
\hline Lymph node metastasis, n (\%) & & & 2.778 & 0.211 \\
\hline Yes & $2(20.0)$ & $8(53.3)$ & & \\
\hline No & $8(80.0)$ & $7(46.7)$ & & \\
\hline Differentiation, n (\%) & & & 6.177 & $0.023^{\mathrm{b}}$ \\
\hline Poor & $5(50.0)$ & $14(93.3)$ & & \\
\hline High/medium & $5(50.0)$ & 1 (6.7) & & \\
\hline TNM stage, n (\%) & & & 7.819 & $0.012^{\mathrm{b}}$ \\
\hline $\mathrm{I}+\mathrm{II}$ & $9(90.0)$ & $5(33.3)$ & & \\
\hline III & $1(10.0)$ & $10(66.7)$ & & \\
\hline Chemotherapy $^{\mathrm{a}}, \mathrm{n}(\%)$ & & & 0.260 & 0.610 \\
\hline Yes & $3(30.0)$ & $6(40.0)$ & & \\
\hline No & $7(70.0)$ & $9(60.0)$ & & \\
\hline Age, years, mean \pm SD & $63.40 \pm 7.21$ & $65.00 \pm 13.30$ & $-0.346^{c}$ & 0.732 \\
\hline White blood cells $\left(\times 10^{9} / \mathrm{L}\right)$, mean \pm SD & $6.47 \pm 1.51$ & $6.32 \pm 1.44$ & $0.255^{\mathrm{c}}$ & 0.801 \\
\hline Total bilirubin, $\mu \mathrm{mol} / 1$, median (interquartile range) & $14.80(10.60,22.10)$ & $21.20(11.50,177.30)$ & $93.500^{\mathrm{d}}$ & 0.311 \\
\hline $\mathrm{CRP}, \mathrm{g} / \mathrm{l}$, median (interquartile range) & $1.90(1.08,3.63)$ & $5.90(2.80,18.80)$ & $121.000^{\mathrm{d}}$ & $0.010^{\mathrm{b}}$ \\
\hline CEA, $\mu \mathrm{g} / 1$, median (interquartile range) & $1.97(1.48,3.49)$ & $2.13(1.57,6.31)$ & $87.000^{\mathrm{d}}$ & 0.531 \\
\hline CA199, U/ml, median (interquartile range) & $10.55(8.30,20.79)$ & $109.76(23.21,479.20)$ & $123.000^{\mathrm{d}}$ & $0.007^{\mathrm{b}}$ \\
\hline
\end{tabular}

${ }^{a}$ Oxaliplatin/tegafur combined with gemcitabine chemotherapy after surgery; ${ }^{b} \mathrm{P}<0.05 ;{ }^{\mathrm{c} t}$ value (unpaired Student's $\mathrm{t}$-test); ${ }^{\mathrm{d}} \mathrm{U}$ value (Mann-Whitney U test). GOLPH3, Golgi phosphoprotein 3; GBC, gallbladder cancer; NLRP3, Nucleotide-binding domain leucine-rich repeat and pyrin domain containing receptor 3; SD, standard deviation; CRP, C-reactive protein; CA199, carbohydrate antigen 19-9; CEA, carcinoembryonic antigen.

GOLPH3-overexpressing cells increased by $50 \%$ compared with the 3xFlag group (Fig. 4E). In summary, GOLPH3 overexpression promoted the proliferation of GBC cells.

GOLPH3 regulates the NLRP3/Caspase-1 pathway. It has been reported that GOLPH3 is related to PtdIns(4)p and the mTOR pathway, and that PtdIns(4)p is an important trigger for NLRP3 activation (21). There is also evidence showing that overexpression of mTOR can upregulate the levels of NLRP3 (30). Therefore, whether GOLPH3 could regulate NLRP3 was next assessed. Following knockdown or overexpression of GOLPH3, the protein expression levels of NLRP3 were determined by western blotting. As shown in Fig. $5 \mathrm{~A}$ and $\mathrm{B}$, the expression levels of NLRP3 in the cells transfected with GOLPH3-SiA and $-\mathrm{SiB}$ were significantly reduced, and significantly increased in the GOLPH3-overexpressing cells, suggesting that GOLPH3 could indeed regulate NLRP3. NLRP3 was knocked down in GBC-SD cells using siRNA, and proof of transfection was determined via western blotting (Fig. 5C). The expression levels of GOLPH3 were not significantly altered following knockdown of NLRP3 in GBC-SD cells (Fig. 5D), suggesting that NLRP3 may be located downstream of GOLPH3.

Whether GOLPH3 could activate Caspase-1 was next determined. The results of western blotting showed that overexpression of GOLPH3 increased Caspase-1 P10 levels, whereas knockdown of GOLPH3 reduced Caspase-1 P10 levels (Fig. 5A and B). Additionally, IL-1 $\beta$ and p-IкB, members of the $\mathrm{NF}-\kappa \mathrm{B}$ signaling pathway, showed similar trends to NLRP3 in the experiments (Fig. 5A and B). These results suggested that GOLPH3 could activate Caspase-1 through regulating NLRP3, and may have promoted the proliferation of GBC via the IL- $1 \beta$ and NF- $\kappa$ B pathway.

GOLPH3 promotes GBC cell proliferation partially via regulation of NLRP3 in GBC-SD cells. The aforementioned results indicated that GOLPH3 could increase the levels of NLRP3, as well as Caspase-1 activity, and GOLPH3 could also promote the proliferation of cells in GBC-SD. Thus, whether the effects of GOLPH3 on GBC cells were mediated via an NLRP3/Caspase-1 pathway were determined. The results of the EdU assay (Fig. 6C and D) showed that knockdown of NLRP3 alone inhibited the proliferation of GBC cells, and overexpression of GOLPH3 alone promoted it. However, in the GOLPH3 + NLRP3-SiR co-transfection group, the number of EdU-positive cells decreased, suggesting that the downregulation of NLRP3 partially offset cell proliferation caused by GOLPH3 overexpression in GBC-SD cells. The CCK-8 assays also showed similar results (Fig. 6E). In 

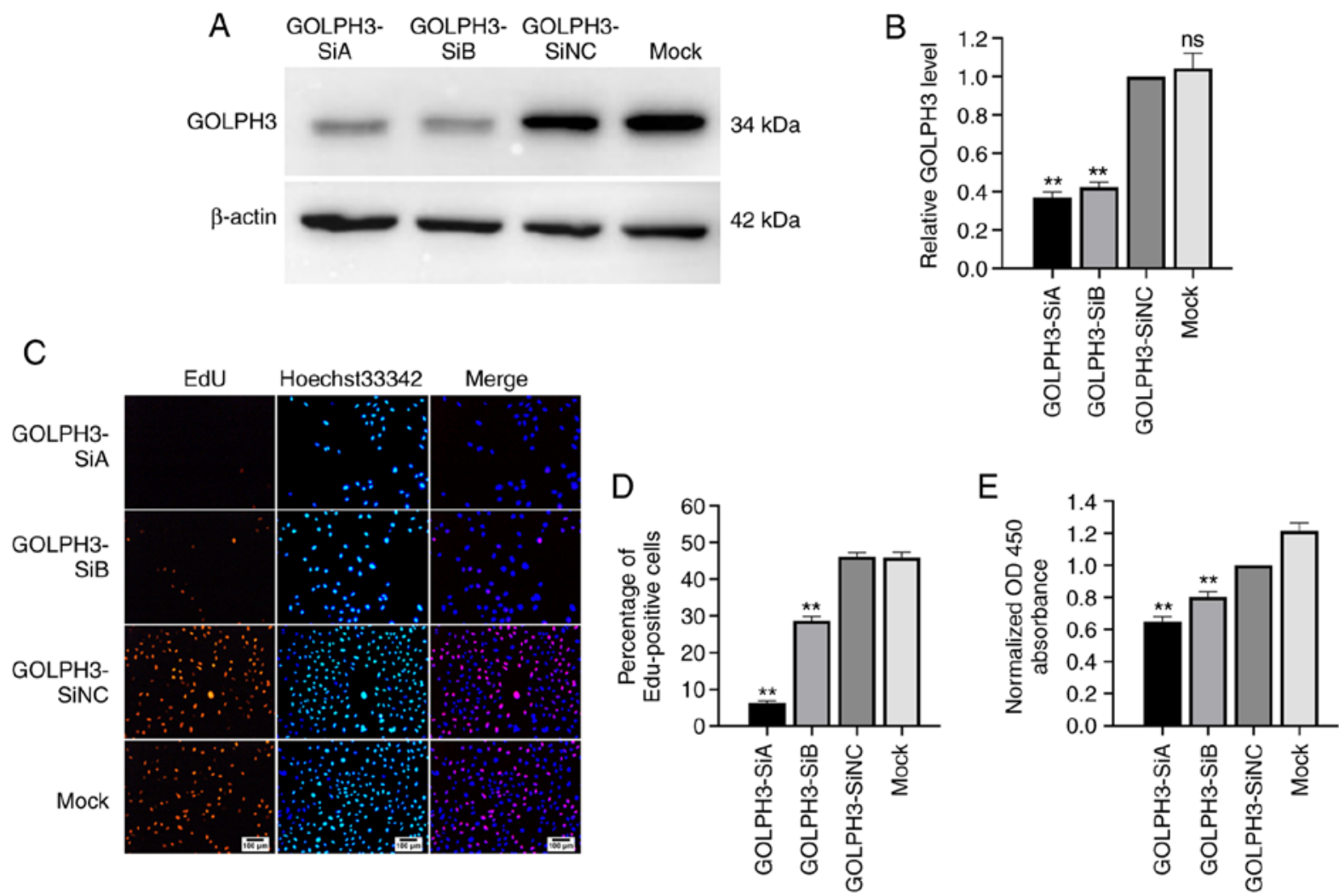

Figure 3. Knockdown of GOLPH3 inhibits the proliferation of GBC cells. (A and B) The silencing efficiency of GOLPH3-SiA and -SiB were examined by western blotting. (C and D) EdU assay showed that knockdown with GOLPH3-SiA and -SiB reduced the proliferation of the GBC-SD cells. (E) Cell Counting Kit- 8 assays showed that GOLPH3-SiA and -SiB inhibited the viability of GBC-SD cells. Data are expressed as the mean \pm standard error of the mean of three independent experiments. Scale bar, $100 \mu \mathrm{m}$. ${ }^{* *} \mathrm{P}<0.01$ vs. GOLPH3-SiNC group. GOLPH3, Golgi phosphoprotein 3; GBC, gallbladder cancer; siRNA/Si, small interfering RNA; NC, negative control; ns, not significant.

addition, the activation of Caspase-1 (pro-caspase-1/Caspase-1 P10) caused by GOLPH3 overexpression was partially abolished by knockdown of NLRP3, suggesting that the activation of Caspase-1 by GOLPH3 was also mediated by NLRP3 (Fig. 6A and B). Taken together, these results suggested that GOLPH3 promoted GBC cell proliferation, at least partially via a NLRP3/Caspase-1 pathway.

\section{Discussion}

In the present study, it was shown that GOLPH3 contributed to GBC progression via upregulation of NLRP3 expression, which resulted in the activation of Caspase-1, IL- $1 \beta$ and the NF- $\mathrm{KB}$ pathway. Additionally, GOLPH3, as well as NLRP3, were both associated with a poor prognosis in patients with GBC, which was supported by the following results. Firstly, knockdown of GOLPH3 inhibited GBC cell proliferation, whereas GOLPH3 overexpression promoted cell proliferation. Secondly, GOLPH3 regulated the protein expression levels of NLRP3 and the activation of Caspase-1, IL-1 $\beta$, as well as the NF- $\mathrm{KB}$ pathway (p-IкB), whereas NLRP3 did not regulate GOLPH3. Thirdly, cell proliferation induced by overexpression of GOLPH3 was partially abolished by GOLPH3 knockdown. Additionally, GOLPH3 and NLRP3 were shown to be highly expressed in human GBC tissues, and there was a significant positive correlation between the expression of these two proteins, and both were positively correlated with the expression of Ki-67 in tissues. Finally, the survival of GOLPH3- or NLRP3-positive patients with GBC was worse than that of cases with negative expression, and GOLPH3 as well as NLRP3 positive expression were associated with the tumor stage, degree of differentiation, depth of invasion, and CA199 and CRP levels.

Several studies have reported that the activation of the NLRP3/Caspase-1 pathway is associated with tumor progression (24-27). Wang et al (25) found that when the NLRP3 inflammasome is activated, Caspase-1 is spliced to further activate the Akt, GSK-3p, ERK1/2 and CREB signaling pathways to promote tumor proliferation. The role of NLRP3 in cancer has gained increasing attention recently. However, the majority of previous studies have focused on investigating the downstream effectors of NLRP3. There has been no reports regarding the upstream effectors of NLRP3 in tumor progression to date, to the best of our knowledge. Conversely, although there is evidence showing that the TGN (21), Ptdins(4)p (21), mTOR (30) and PD2 (31) are closely related to the activation of NLRP3, these studies were all performed in macrophages. Whether this relationship also exists in tumor cells, and which proteins regulate these interactions remains unclear. In addition, several studies have reported that GOLPH3 is involved in the progression of various malignant tumors, such as lung cancer, pancreatic cancer, gastric cancer and brain glioma, via different signaling pathways, including the mTOR and FOXO1 pathway (6,11-14). However, the role of GOLPH3 in the progression of GBC is yet to be elucidated. 

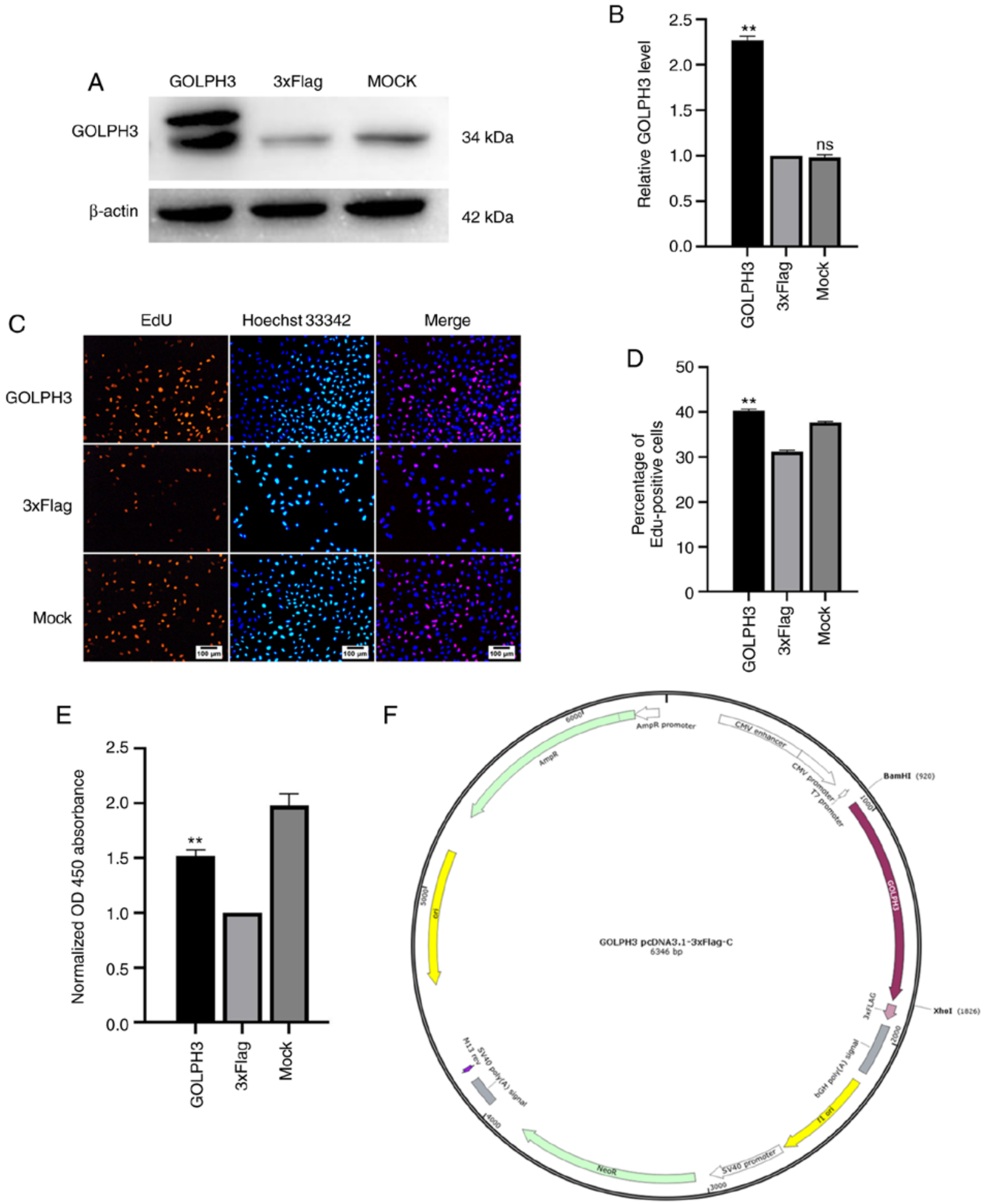

Figure 4. Overexpression of GOLPH3 promotes the proliferation of GBC cells. (A and B) Western blotting was performed to determine the transfection efficiency of GOLPH3-overexpression vector in the GBC-SD cells. (C and D) EdU assays showed that overexpression of GOLPH3 promoted the proliferation of GBC-SD cells. (E) Cell Counting Kit-8 assays showed that overexpression of GOLPH3 promoted the viability of GBC-SD cells. (F) Map of the GOLPH3 pcDNA-3xFlag-C plasmid. Data are expressed as the mean \pm standard error of the mean of three independent experiments. Scale bar, $100 \mu \mathrm{m} .{ }^{* *} \mathrm{P}<0.01 \mathrm{vs}$. 3xFlag group. GOLPH3, Golgi phosphoprotein 3; GBC, gallbladder cancer; ns, not significant.

In the present study, GOLPH3 and NLRP3 were shown to be highly upregulated in clinical human GBC tissues, and there was a positive correlation between these two molecules, and both were also positively correlated with the expression of Ki-67 in the GBC tissues. Moreover, GOLPH3 promoted the proliferation of GBC cells and regulated the expression of NLRP3 as well as the activation of Caspase-1. However, NLRP3 did not affect GOLPH3 levels, indicating that GOLPH3 was located upstream of NLRP3. NLRP3 can activate Caspase-1, and further activate the Akt, GSK-3p, ERK1/2 and CREB pathways to promote adenocarcinoma proliferation (25). In the present study, it was shown that not 


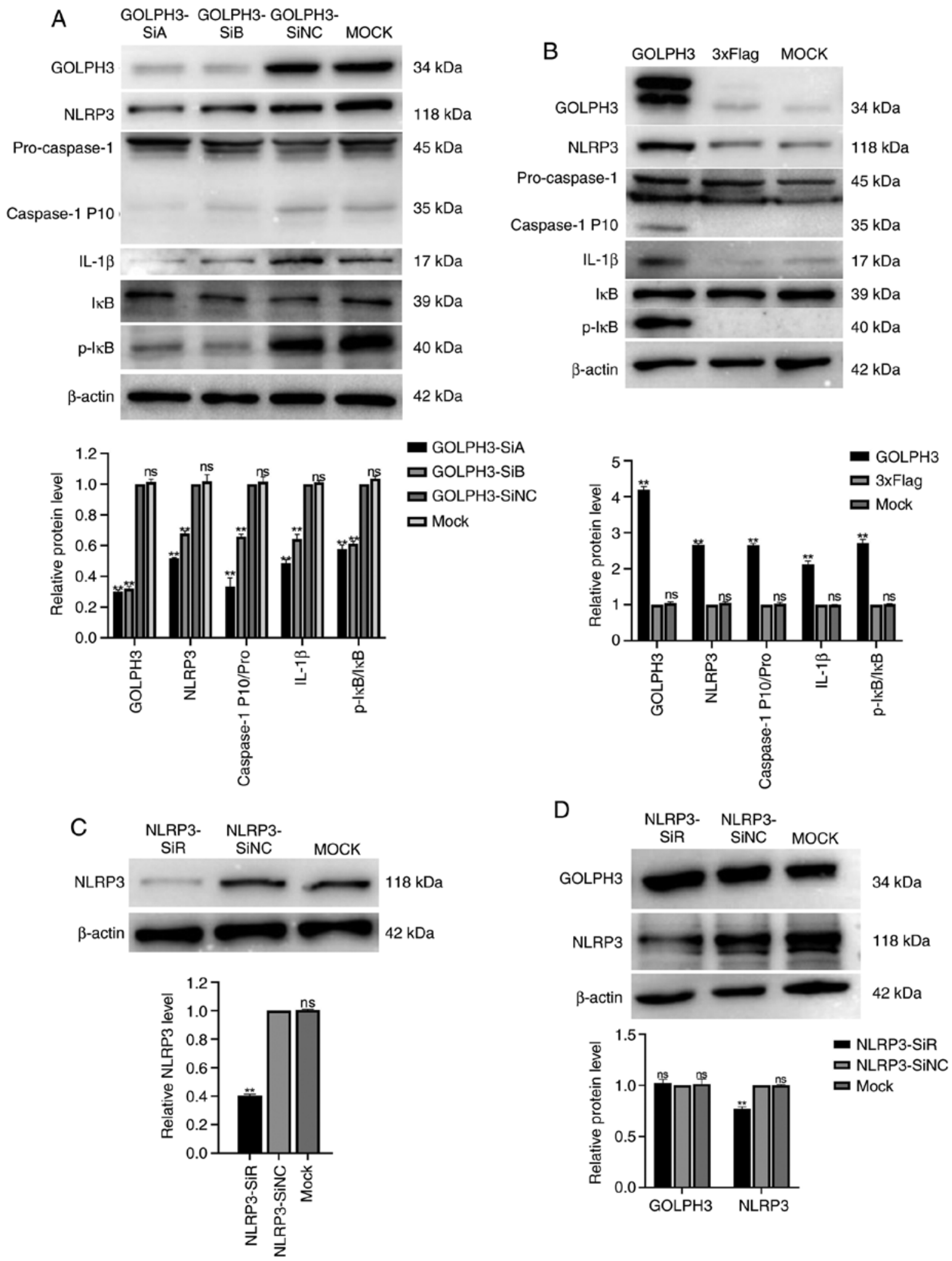

Figure 5. GOLPH3 regulates NLRP3. (A) GOLPH3 knockdown decreased the levels of NLRP3 and inhibited Caspase-1 activity (Caspase-1 P10), decreased

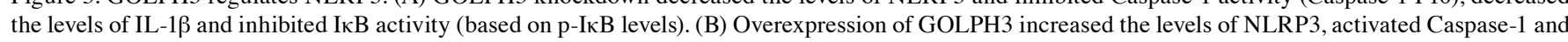
increased the levels of IL-1 $\beta$ and activated IкB. (C) The silencing efficiency of NLRP3 siRNA were examined by western blotting. (D) NLRP3 knockdown had no effect on the levels of GOLPH3. Data are expressed as the mean \pm standard error of the mean of three independent experiments. ** P $<0.01$ vs. GOLPH3-SiNC group. GOLPH3, Golgi phosphoprotein 3; p-, phosphorylated; NLRP3, nucleotide-binding domain leucine-rich repeat and pyrin domain containing receptor 3; siRNA/Si, small interfering RNA; NC, negative control; ns, not significant.

only NLRP3, but also GOLPH3 could promote the activation of Caspase-1, and this activation could be offset by knocking down NLRP3. Additionally, as IL-1 $\beta$, an important member of NLRP3 inflammasome, has been reported to be associated with tumor proliferation, and the NF- $\mathrm{KB}$ signaling pathway is upstream of the inflammasome $(21,25,28)$, the levels of IL-1 $\beta$ 

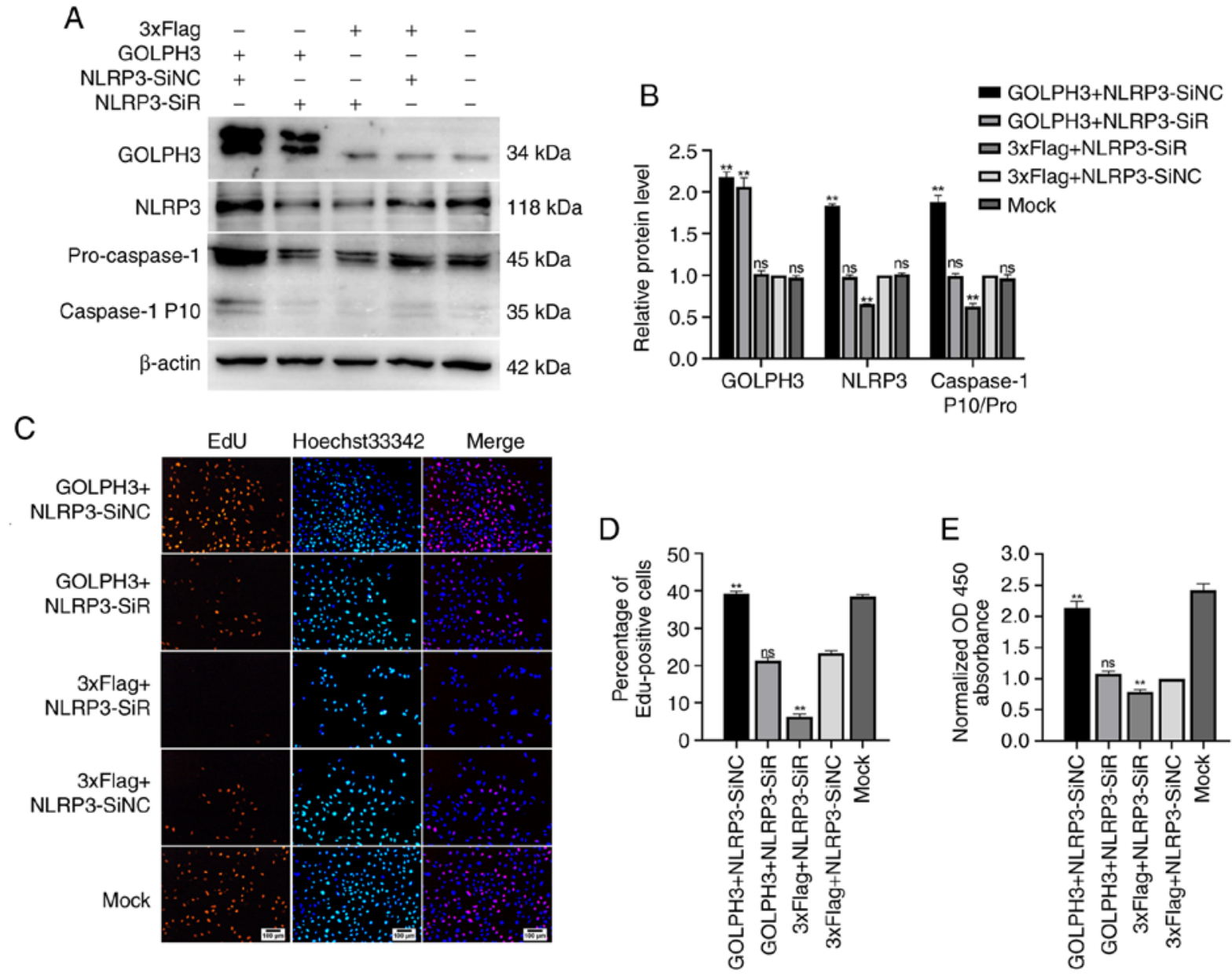

Figure 6. GOLPH3 promotes the proliferation of GBC cells by regulating NLRP3 in GBC-SD cells. (A and B) Western blotting was used to detect the efficiency of co-transfection of GOLPH3 + NLRP3-SiR. (C and D) EdU assays showed that cell proliferation induced by overexpression of GOLPH3 was inhibited by NLRP3-SiR. (E) Cell Counting Kit-8 assays showed that cell viability induced by overexpression of GOLPH3 was reduced by NLRP3-SiR. Data are expressed as the mean \pm standard error of the mean of three independent experiments. Scale bar, $100 \mu \mathrm{m} .{ }^{* *} \mathrm{P}<0.01 \mathrm{vs}$. 3xFlag + NLRP3-SiNC group. GOLPH3, Golgi phosphoprotein 3; GBC, gallbladder cancer; ns, not significant; siRNA/Si, small interfering RNA; NLRP3, nucleotide-binding domain leucine-rich repeat and pyrin domain containing receptor 3 ; $\mathrm{NC}$, negative control.

and $\mathrm{NF}-\kappa \mathrm{B}$ pathway activation (based on $\mathrm{p}-\mathrm{I} \kappa \mathrm{B}$ levels) were determined. The results showed that GOLPH3 could also regulate the protein expression levels of IL-1 $\beta$ and the activation of $\mathrm{I} \kappa \mathrm{B}$, which indicated that GOLPH3 may promote the proliferation of GBC via IL- $1 \beta$ and the NF- $\kappa B$ pathway. Thus, GOLPH3 can also regulate the expression of NLRP3, further activate Caspase-1, IL-1 $\beta$ and the $\mathrm{NF}-\kappa \mathrm{B}$ pathway to promote the proliferation of gallbladder adenocarcinoma cells.

As for the exact mechanism by which GOLPH3 regulates NLRP3, this remains unknown. Based on the existing research, it is hypothesized that on the one hand, the excessive activation of GOLPH3 may cause the fragmentation of the TGN and break the binding of GOLPH3 and PtdIns(4) p. The free PtdIns(4)p then bind to NLRP3 and activates the entire inflammasome, further affecting the protein levels of NLRP3 (8,21). Additionally, GOLPH3 activates the mTOR molecular pathway, which further increases the levels of NLRP3 (30). However, due to a lack of direct and effective methods to detect the activation of NLRP3, only the levels of NLRP3 expression were assessed in the present study. It is unclear whether the effects of GOLPH3 on NLRP3 occur by affecting the activity of NLRP3, or by directly affecting the expression of NLRP3. It will be interesting to further reveal the underlying mechanism by which GOLPH3 regulates NLRP3.

In addition, through the analysis of patient clinical data, it was found that patients with positive expression of GOLPH3 or NLRP3 had worse overall survival compared with the negative patients, suggesting that this pathway also affects the overall survival of patients in vivo. Additionally, GOLPH3and NLRP3-positive patients had less differentiated tumors, more advanced tumors, deeper infiltration and high levels of CA199, suggesting that the tumors in these patients were more aggressive compared with the negative patients. Of note, it was found that the expression of GOLPH3 and NLRP3 in vivo was associated with the levels of CRP in the peripheral blood, which further revealed the connection between this pathway and inflammation.

In conclusion, it was observed that GOLPH3 promoted the proliferation of GBC cells by regulating NLRP3 and further activating Caspase-1, IL-1 $\beta$ and the NF- $\kappa$ B pathway in vitro. Additionally, the positive correlation between the expression levels of these proteins was shown, and it was also shown that their expression was positively correlated with the expression of the proliferation marker Ki-67 in vivo. Patients with 
positive expression of GOLPH3 or NLRP3 tended to have worse overall survival, and the expression of both of these proteins was related to the patients' tumor stage, degree of differentiation, depth of invasion, and CA199 and CRP levels. This research provided evidence regarding the existence of a GOLPH3-NLRP3-Caspase-1 signaling pathway in human GBC.

\section{Acknowledgements}

Not applicable.

\section{Funding}

No funding was received.

\section{Availability of data and materials}

All data generated or analyzed during this study are included in this published article.

\section{Authors' contributions}

KL contributed to the conception of the present study.ZZ prepared the manuscript and contributed to performing the experiments. $\mathrm{KL}$ and $\mathrm{ZZ}$ confirm the authenticity of all the raw data. QZ analyzed the data and performed part of the experiments. DC taught the laboratory techniques needed in the present study and assisted in completing part of the experiments. LC contributed to designing the experiments. LC and WX contributed to the manuscript editing. YB contributed to data collection. WX performed the follow-ups of patients in the present study. All authors read and approved the final manuscript.

\section{Ethics approval and consent to participate}

Written informed consent was obtained from all patients and the study was approved by the Ethics Committee of the 904th Hospital of Joint Logistic Support Force of PLA (approval no. 2019-10-006; Wuxi, China).

\section{Patient consent for publication}

Not applicable.

\section{Competing interests}

The authors declare that they have no competing interests.

\section{References}

1. Hundal R and Shaffer EA: Gallbladder cancer: Epidemiology and outcome. Clin Epidemiol 6: 99-109, 2014.

2. Gamboa AC and Maithel SK: The landmark series: Gallbladder cancer. Ann Surg Oncol 27: 2846-2858, 2020.

3. Song X, Hu Y, Li Y, Shao R, Liu F and Liu Y: Overview of current targeted therapy in gallbladder cancer. Signal Transduct Target Ther 5: 230, 2020.

4. Goetze TO: Gallbladder carcinoma: Prognostic factors and therapeutic options. World J Gastroenterol 21: 12211-12217, 2015.

5. Kuna RS and Field SJ: GOLPH3: A Golgi phosphatidylinositol(4) phosphate effector that directs vesicle trafficking and drives cancer. J Lipid Res 60: 269-275, 2019.
6. Zhou X, Xue P, Yang M, Shi H, Lu D, Wang Z, Shi Q, Hu J, Xie S, Zhan W and Yu R: Protein kinase D2 promotes the proliferation of glioma cells by regulating Golgi phosphoprotein 3. Cancer Lett 355: 121-129, 2014

7. Sechi S, Frappaolo A, Karimpour-Ghahnavieh A, Piergentili R and Giansanti MG: Oncogenic roles of GOLPH3 in the physiopathology of cancer. Int J Mol Sci 21: 933, 2020.

8. Farber-Katz SE, Dippold HC, Buschman MD, Peterman MC, Xing M, Noakes CJ, Tat J, Ng MM, Rahajeng J, Cowan DM, et al: DNA damage triggers Golgi dispersal via DNA-PK and GOLPH3. Cell 156: 413-427, 2014.

9. Buschman MD, Xing M and Field SJ: The GOLPH3 pathway regulates Golgi shape and function and is activated by DNA damage. Front Neurosci 9: 362, 2015.

10. Bergeron JJM, Au CE, Thomas DY and Hermo L: Proteomics identifies Golgi phosphoprotein 3 (GOLPH3) with a link between Golgi structure, cancer, DNA damage and protection from cell death. Mol Cell Proteomics 16: 2048-2054, 2017.

11. Tang S, Pan H, Wei W, Yang H, Liu J and Yang R: GOLPH3: A novel biomarker that correlates with poor survival and resistance to chemotherapy in breast cancer. Oncotarget 8: 105155-105169, 2017.

12. Liu H, Wang X, Feng B, Tang L, Li W, Zheng X, Liu Y, Peng Y, Zheng $\mathrm{G}$ and $\mathrm{He}$ Q: Golgi phosphoprotein 3 (GOLPH3) promotes hepatocellular carcinoma progression by activating mTOR signaling pathway. BMC Cancer 18: 661, 2018.

13. Liu J, Wei H, Lai L, Wang Y, Han X and Zhang Z: Golgi phosphoprotein-3 promotes invasiveness of gastric cancer cells through the mTOR signalling pathway. Clin Invest Med 42: E38-E47, 2019.

14. Zhao C, Zhang J, Ma L, Wu H, Zhang H, Su J, Geng B, Yao Q and Zheng J: GOLPH3 promotes angiogenesis of lung adenocarcinoma by regulating the $\mathrm{Wnt} / \beta$-catenin signaling pathway. Onco Targets Ther 13: 6265-6277, 2020.

15. Zhang Q, Zhuang J, Deng Y, Yang L, Cao W, Chen W, Lin T, Lv X, Yu H, Xue Y and Guo H: miR34a/GOLPH3 axis abrogates urothelial bladder cancer chemoresistance via reduced cancer stemness. Theranostics 7: 4777-4790, 2017.

16. Yu T, An Q, Cao XL, Yang H, Cui J, Li ZJ and Xiao G: GOLPH3 inhibition reverses oxaliplatin resistance of colon cancer cells via suppression of PI3K/AKT/mTOR pathway. Life Sci 260: 118294, 2020.

17. Zeng Z, Lin H, Zhao X, Liu G, Wang X, Xu R, Chen K, Li J and Song L: Overexpression of GOLPH3 promotes proliferation and tumorigenicity in breast cancer via suppression of the FOXO1 transcription factor. Clin Cancer Res 18: 4059-4069, 2012.

18. Lim Y and Kumar S: A single cut to pyroptosis. Oncotarget 6: 36926-36927, 2015.

19. Islam MT, Bardaweel SK, Mubarak MS, Koch W, Gaweł-Beben K, Antosiewicz B and Sharifi-Rad J: Immunomodulatory effects of diterpenes and their derivatives through NLRP3 inflammasome pathway: A review. Front Immunol 11: 572136, 2020.

20. Latz E, Xiao TS and Stutz A: Activation and regulation of the Inflammasomes. Nat Rev Immunol 13: 397-411, 2013.

21. Chen J and Chen ZJ: PtdIns4P on dispersed trans-Golgi network mediates NLRP3 inflammasome activation. Nature 564: 71-76, 2018.

22. Feng YS, Tan ZX, Wu LY, Dong F and Zhang F: The involvement of NLRP3 inflammasome in the treatment of Alzheimer's disease. Ageing Res Rev 64: 101192, 2020.

23. Lu F, Zhao Y, Pang Y, Ji M, Sun Y, Wang H, Zou J, Wang Y, Li G, Sun T, et al: NLRP3 inflammasome upregulates PD-L1 expression and contriwhilees to immune suppression in lymphoma. Cancer Lett 497: 178-189, 2021.

24. Tezcan G, Garanina EE, Zhuravleva MN, Hamza S, Rizvanov AA and Khaiboullina SF: Rab GTPase mediating regulation of NALP3 in colorectal cancer. Molecules 25: 4834, 2020.

25. Wang Y, Kong H, Zeng X, Liu W, Wang Z, Yan X, Wang H and Xie W: Activation of NLRP3 inflammasome enhances the proliferation and migration of A549 lung cancer cells. Oncol Rep 35: 2053-2064, 2016.

26. Chen Z, Liu Q, Zhu Z, Xiang F, Zhang M, Wu R and Kang X: Ursolic acid protects against proliferation and inflammatory response in LPS-Treated gastric tumour model and cells by inhibiting NLRP3 inflammasome activation. Cancer Manag Res 12: 8413-8424, 2020.

27. Chen Z, He M, Chen J, Li C and Zhang Q: Long non-coding RNA SNHG7 inhibits NLRP3-dependent pyroptosis by targeting the miR-34a/SIRT1 axis in liver cancer. Oncol Lett 20: 893-901, 2020. 
28. Guo R, Qin Y, Shi P, Xie J, Chou M and Chen Y: IL-1 $\beta$ promotes proliferation and migration of gallbladder cancer cells via Twist activation. Oncol Lett 12: 4749-4755, 2016.

29. DippoldHC,NgMM,Farber-KatzSE,LeeSK,KerrML,PetermanMC, Sim R, Wiharto PA, Galbraith KA, Madhavarapu S, et al: GOLPH3 bridges phosphatidylinositol-4-phosphate and actomyosin to stretch and shape the Golgi to promote budding. Cell 139: 337-351, 2009.

30. Li X, Zhang X, Pan Y, Shi G, Ren J, Fan H, Dou H and Hou Y: mTOR regulates NLRP3 inflammasome activation via reactive oxygen species in murine lupus. Acta Biochim Biophys Sin (Shanghai) 50: 888-896, 2018.

31. Zhang Z, Meszaros G, He WT, Xu Y, de Fatima Magliarelli H, Mailly L, Mihlan M,Liu Y,Puig Gámez M, Goginashvili A, et al: Protein kinase D at the Golgi controls NLRP3 inflammasome activation. J Exp Med 214: 2671-2693, 2017.
32. Friedrichs K, Gluba S, Eidtmann $\mathrm{H}$ and Jonat W: Overexpression of p53 and prognosis in breast cancer. Cancer 72: 3641-3647, 1993.

33. Lamartina L, Grani G, Arvat E, Nervo A, Zatelli MC, Rossi R, Puxeddu E, Morelli S, Torlontano M, Massa M, et al: 8th edition of the AJCC/TNM staging system of thyroid cancer: What to expect (ITCO\#2). Endocr Relat Cancer 25: L7-L11, 2018.

34. Hui AM, Shi YZ, Li X, Sun L, Guido T, Takayama T and Makuuchi M: Proliferative marker Ki-67 in gallbladder carcinomas: High expression level predicts early recurrence after surgical resection. Cancer Lett 176: 191-198, 2002.

This work is licensed under a Creative Commons Attribution-NonCommercial-NoDerivatives 4.0 International (CC BY-NC-ND 4.0) License. 